\title{
Broadcasting in LTE-Advanced networks using multihop D2D communications
}

\author{
Giovanni Nardini $^{(1)}$, Giovanni Stea ${ }^{(1)}$, Antonio Virdis ${ }^{(1)}$, Dario Sabella ${ }^{(2)}$, Marco Caretti ${ }^{(2)}$ \\ (1) Dipartimento di Ingegneria dell'Informazione, University of Pisa \\ Largo Lucio Lazzarino 1, I-56122, Pisa, Italy \\ g.nardini@ing.unipi.it, giovanni.stea@unipi.it, a.virdis@iet.unipi.it \\ (2) Telecom Italia Lab, Turin, Italy \\ \{dario.sabella,marco.caretti\}@telecomitalia.it
}

\begin{abstract}
In an LTE-Advanced network, network-controlled Device-to-Device (D2D) communications can be combined in a multihop fashion to distribute broadcasts over user-defined (and possibly large) areas, with small latencies and occupying few resources. Such a service may be exploited for several purposes, (e.g. Internet of Things, Vehicular communications). Engineering a multihop D2D-based broadcast service requires working at both the application level on the User Equipment (UE) and at the resource-allocation level within the eNodeBs. This paper describes the necessary modifications at both the UE and the eNodeB, what the main issues are, and how to solve them efficiently. We evaluate the performance of the above service using system-level simulations, and demonstrate its advantages over standard broadcasting techniques.
\end{abstract}

Keywords-LTE-Advanced, LTE-A, device-to-device, multihop, resource allocation

\section{INTRODUCTION}

Proximity-based broadcast over device-to-device (D2D) transmissions is expected to foster a number of value-added services, such as advertising, smart-city applications, etc. [1]. There are applications, however, that need to broadcast their messages over larger radiuses than a single D2D transmission's. Typical cases are vehicular or robotics collision alerts or Distributed Hash Table (DHT) lookup requests in Internet-ofThings deployments [2]. These messages must be propagated in a well-defined area, which may or may not match a single cell (it can be both a portion of a cell, or encompass more than one cell). Furthermore, the geographical area must be covered in a short time, either because of a specific deadline, or because the performance of applications relying on these broadcast messages depends on how fast these propagate.

To address the above issues, an obvious possibility is to have the eNB act as a relay: the UE that generates the message sends it to the eNB, which in turn relays it to the UEs on the required area, fast and reliably. This can be done either using the built-in multicast/broadcast facilities of LTE (i.e., Multicast/Broadcast SubFrame Network, MBSFN), or using unicast transmissions in the downlink. Unfortunately, both options have major drawbacks. eNB-level multicast, in fact, carries at least three problems: the first one is that multicast/broadcast subframes can be used exclusively for that purpose and they must be declared semi-statically. This means that the reactiveness to (e.g.) an infrequent alert must be traded against the periodic and non-negligible associated loss of downlink unicast capacity. For instance, if the network declares one subframe per frame to be an MBSFN one, it loses $10 \%$ of unicast capacity, and still has a worst-case delay of $10 \mathrm{~ms}$ to relay an alert. The second problem is that the above mechanism does not allow to fine-tune the geographical reach: in fact, MBSFN must be declared across a tracking area, which may encompass more than one cell, and all the UEs in that tracking area will hear the message. There is no way to keep the message confined to a smaller portion of a tracking area. The third and last problem is that multicasting at the eNB requires that the latter chooses - once and for all recipients - a transmission format: thus, some of the UEs that are in the target area may not be able to decode the message, which hampers reliability.

If, instead, the eNB relays the message using unicast transmissions, we avoid the problem of MBSFN inflexibility and unreliability, and of geographical span as well, assuming that the eNB knows the positions of the intended recipients (an assumption which becomes more likely with the progress of localization services). However, a simple computation shows that this may require an inordinate amount of resources, especially in dense networks. Assume that a 40-byte message has to be sent to 100 UEs using unicast transmissions. If the mean Channel Quality Indicator (CQI) is 5, then three Resource Blocks (RBs) per UE are needed on average, which means that three entire subframes are required to send the message to all of them. The cost in terms of exploited capacity and energy (and, possibly, service disruption for other users) is thus non negligible.

This paper exploits a different possibility, which - to the best of our knowledge - has not been considered so far, i.e. leveraging multihop point-to-multipoint D2D transmissions: the latter do not increase the operator's energy bill, since they are scheduled by the eNB on the sidelink (which is often physically allocated in the UL frame, [4]). Moreover, D2D transmission may also leverage frequency reuse, which makes them quite economical in terms of resource consumption, and - as we show in this paper - the latency involved in covering relatively large broadcast radiuses is tolerable. To run broadcasts on multihop $\mathrm{D} 2 \mathrm{D}$, some intelligence is required on the UE at the application level, in order to decide when to relay a message and mitigate 


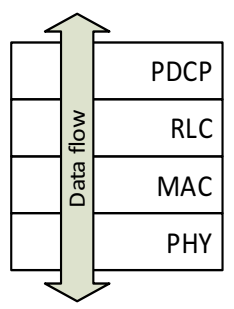

Fig. 1. LTE-A protocol stack

collisions. Moreover, the eNBs must allocate resources, either statically or dynamically, and possibly in a coordinated way across neighboring cells, for this to happen.

This paper describes the modifications required within the UE and the eNB to enable the above services, discusses the options for each of these and weighs its pros and cons, and finally evaluates its performance in terms of latency, resource consumption and effectiveness. Similar broadcast diffusion problems have been addressed in other contexts, especially in infrastructureless wireless networks such as ad hoc or sensor ones (e.g., [12]-[13]). However, the fact that in LTE resources are scheduled by a central entity, and can be accessed on demand, makes this setting quite different. The above problem also bears some resemblance to the one of resource allocation (i.e., channel assignment and/or link scheduling) in Wireless Mesh Networks. For the above, centralized decision-making is sometimes assumed, although far less often than distributed decision-making, (see, e.g., [5]-[6]). The settings are however quite different, in that it is assumed that nodes are equipped with a small number of radios, which can be tuned to a (larger, but still limited) number of non-interfering channels. In our case, all UEs have as many "radios" and "channels" as the number of RBs, which is in the order of several tens. Moreover, the algorithms presented in the literature often assume periodic transmissions and long-term, semi-static resource allocations, and unicast point-to-point transmissions. Our broadcasting problem cannot be accommodated using these algorithms. Finally, some works do advocate multihop D2D transmissions in LTE-A (e.g., [15]-[17]). However, these deal with one-to-one transmissions, and do not consider broadcast.

The rest of the paper is organized as follows: Section II describes the background and hypotheses. Section III presents our contribution, which is evaluated in Section IV. Section V concludes the paper.

\section{BACKGROUND AND SYSTEM MODEL}

This section describes the LTE-A protocol stack and introduces point-to-multipoint (P2MP) D2D communications.

The LTE-A protocol stack is located at level 2 of the OSI model and it consists of the four layers depicted in Fig. 1. Starting from the top, the Packet Data Convergence Protocol (PDCP) receives IP datagrams, cyphers them and sends them down to the Radio Link Control (RLC) layer, which stores them in the form of RLC SDUs. When the underlying MAC layer requests data for transmission, the RLC behaves accordingly to its configured mode, i.e. transparent (TM), unacknowledged (UM) or acknowledged (AM). TM does not perform additional

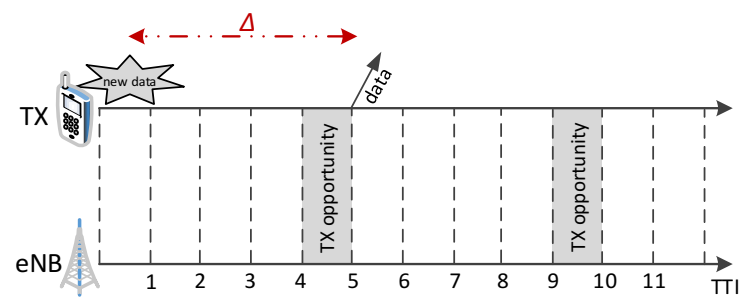

Fig. 3. Autonomous Resource Selection

operations. UM segments/concatenates RLC SDUs to fit the size requested by the MAC. On reception, it reassembles, detects duplicates and reorders RLC PDUs. AM adds an ARQ retransmission mechanism on top of those functionalities. The MAC assembles the RLC PDU(s) into a Transmission Block (TB) by adding its own header, and sends it down to the PHY layer for transmission.

Resource allocation is performed by the eNB's MAC layer on each Transmission Time Interval (TTI) of $1 \mathrm{~ms}$. The available bandwidth can be represented as a vector of Resource Blocks (RBs), which have to be allocated to backlogged UEs according to some scheduling policy. The number of RBs required to send a TB depends on the Signal to Interference and Noise Ratio (SINR) perceived by the UE. To this aim, the UE periodically reports a representation of the SINR in the form of a CQI. The latter is used by the eNB to select the Modulation and Coding Scheme (MCS) for the UE, hence the number of bits that one RB can carry. In the downlink (DL), the eNB sends the TB to a UE on the allocated RBs. In the uplink (UL), the eNB sends a transmission grant to the UE, which specifies which RBs the UE can use to carry its TB, using which transmission format. In order to inform the eNB about the presence of UL traffic, a UE transmit a Buffer Status Report (BSR). However, this can be done if the UE already has some RBs allocated to it, whose space is large enough to contain a BSR. Otherwise, the UE needs to start a Random Access Procedure (RAC), so that the eNB can issue a transmission grant for the BSR in a future TTI. Collisions may occur if simultaneous RAC requests from different UEs use the same resources. Hence, UEs that do not receive a reply within the expected time window re-iterate the requests after a backoff.

Network-controlled D2D communications for LTE-A are being standardized by the 3rd Generation Partnership Project (3GPP), mainly focusing on point-to-multipoint (or one-tomany) communications [3]. D2D enables direct communication between UEs when they are in proximity, without using the traditional two-hop path through the eNB. The new D2D link is also referred to as sidelink (SL). In a Frequency Division Duplex (FDD) system, SL communications usually occurs in the UL spectrum, which is likely to be less loaded than the DL one. In this configuration, D2D-enabled UEs need to be equipped with a Single-Carrier Frequency Division Multiple Access (SC-FDMA) receiver [4]. Resource allocation is still carried out by the eNB according to two possible modes, namely the scheduled resource allocation (SRA) and the autonomous resource selection (ARS). In SRA, the UE sends a $\mathrm{RAC}$ requests to the eNB, followed by a BSR. Then, the eNB schedules resources according to the BSR and communicates its 
decision to the UE. BSR reporting is similar to that of the UL case. This is shown in Fig. 2, along with its timing. In ASR, the UE selects resources from a pool autonomously. The resource pool can be configured by the eNB (semi-)statically, e.g. $M$ RBs every $T$ TTIs. With reference to Fig. 3, the UE has new data to transmit at $t=1$, but it needs to wait for the next eligible TTI, i.e. at $t=5$. Collisions may occur in this last case, if more than one UE selects the same resources. It is worth remarking that P2MP D2D communications are unacknowledged, hence there is no way for the sender to know which neighboring UE did receive a message, and $\mathrm{H}-\mathrm{ARQ}$ is disabled.

In the following, we consider a LTE-A system where D2Denabled UEs are located in a multicell network. Each UE runs an application that may generate messages (e.g. vehicular collision alerts) destined to all UEs within an arbitrary broadcasting area. Our problem is to reach possibly all the UEs in the broadcasting area, and as few as possible outside it, using only P2MP DL transmissions, possibly relayed by UEs themselves. Fig. 4 exemplifies the system model. The shaded UE generates a message to be delivered to all UEs within the area defined by the circle. Solid arrows represent the first P2MP D2D transmission, and dashed ones represent the relaying from the UEs that successfully received the first transmission. A UE that receives more than one message in the same TTI will only attempt to decode the one received with the strongest power.

Fig. 4. System model

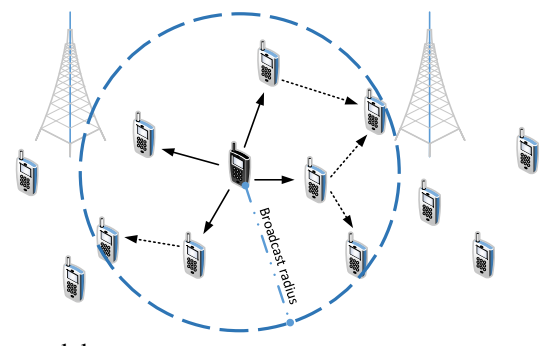

\section{MULTIHOP D2D BROADCASTING}

In multihop D2D broadcast, the eNB does not directly participate in the data plane transmission, i.e. it does not send any data packet, not even using MBSFN or unicast DL transmissions. Instead, the data is broadcast by the applications running on the UEs. However, the eNB is still in control of the resource allocation, which does affect the performance of the broadcasting. Hereafter, we first discuss the application-level issues that need to be addressed at the UEs to support effective broadcasting, and then compare allocation policies at the eNB.

\section{A. Broadcast management within the UE}

The two main problems to be addressed at the UE are how to define a broadcast area and when to relay D2D communications. We first argue that the definition of the broadcast area depends on both the scenario and the type of information: considering a vehicular use case as example, collision notifications may interest vehicles in a radius of few hundred meters, whereas traffic advertisements likely need to reach larger distances to allow drivers to possibly change their route. For the above reasons, this information should be embedded in the application-level message. We identify two options to do this in practice. The simplest approach is to use a
Time-to-live (TTL) mechanism: the source UE sets the TTL field in the application-level packet to the maximum number of hops. That field is decreased by one on reception, and relaying occurs if TTL $>0$. However, this way the broadcast area ends up depending on the network topology, i.e. the UEs' position and density, which may be unpredictable and may change over time. An alternative approach consists in embedding the broadcast area directly in the message, e.g., as the coordinates of the source UE and a maximum broadcast radius. Receiving UEs then check whether their own position falls within the broadcast area before relaying the message. This allows finer tuning of the dimensions and shape of the broadcast area, which is made independent of the UEs' density. On the other hand, it actually defines which UEs relay (as opposed to receive) the message. The actual broadcast reception area exceeds the defined one by up to one UE transmission radius. Moreover, adding location information to the message increases its size, consuming more network resources for transmission. The other key aspect is how to make relaying efficient. Again, this can only happen if relaying occurs at the application level. If UEs blindly relay each received message, an inordinate amount of data may congest the network, possibly disrupting the service for other users. An obvious countermeasure is to employ a suppression mechanism similar to that used by the Trickle algorithm [7]. Trickle is used in Wireless Sensor Networks to regulate the broadcasting of updates and/or routing information. The algorithm can be adapted so that a UE that receives a new message waits for a random time in the range $[I / 2, I), I$ being the Trickle interval. If the same message is received more than $K$ times in the above time window, the UE disables the relaying of that message henceforth.

Finally, it must be considered that P2MP transmissions reach several UEs simultaneously, hence may trigger a storm of quasi-simultaneous relaying attempts. These will in turn generate collisions, whatever the allocation scheme at the eNB (see next section for details). These can be mitigated by delaying UE relaying by a random time in $[0, \Delta), \Delta$ being a configurable parameter. Obviously, the probability of collisions is reduced at the expenses of increasing the latency.

\section{B. Resource allocation in the network}

As already stated, resources are controlled by the eNBs, using either SRA or ARS. In this subsection we discuss the pros and cons of each approach when used in conjunction with multihop relaying, possibly across cell borders.

The first metric to be considered is latency: SRA incurs the overhead of one RAC handshake per transmission, which means $10 \mathrm{~ms}$ delay at each hop at least (see Fig. 2). Note that this is the best-case scenario, since the delay may be longer if a RAC collision occurs or scheduling delay is experienced, e.g. in case of heavy-load conditions. ASR, instead, allows UEs to transmit as soon as a transmission opportunity becomes available, avoiding the RAC/BSR handshake. In this case, the worst-case delay to access the medium is the period $T$. In principle, ASR allocation with small periods may result in faster broadcasting. On the other hand, dedicating large shares to P2MP D2D transmissions hampers UL communications and wastes resources when there is no SL traffic. 
Another key aspect is that of collisions. With SRA, RAC requests may collide at the eNB. However, this is relatively unlikely: in fact, the standard mandates that UEs select at random one in 64 RAC preambles, and requests on different preambles do not collide. Moreover unanswered RAC requests (either due to collision or to lack of response by the eNB) are reiterated after a random backoff time. Thus, RAC collisions do delay the broadcast process, but also desynchronize relaying UEs, hence have positive side effects. Data transmission on the SL is instead made interference-free by the eNB scheduling, possibly exploiting some frequency reuse scheme (such as the one in [10]) that allow faraway UEs to use the same RBs to relay a message. With ARS allocation, instead, data transmission on the SL from different UEs occurs simultaneously on the same RBs, thus possibly causing severe interference at the receiving UEs. This can be mitigated only by increasing the number $M$ and having UEs select randomly which RBs to use. Note that the fact that allocation is periodic forces synchronization among groups of UEs, hence reinforces the occurrence of collisions at each successive hop. A sending UEs cannot know if collision has occurred, so the only possible countermeasure is to repeat the transmission more than once.

As anticipated in Section II, the broadcasting area may encompass several adjacent cells, as shown in Fig. 5. In this scenario, SL transmissions in one cell may occur on resources that are instead used for other transmissions (e.g. standard UL communications) in the neighboring cell, thus preventing its cell-border UEs from correctly receiving the message. Hence, coordination among eNBs is needed. The type of coordination depends on how resources are allocated. If ARS allocation is used, it is sufficient that neighboring eNBs agree on the same allocation pattern. For SRA, more flexible and dynamic coordination algorithms can be applied, possibly exploiting the $\mathrm{X} 2$ interface. For example, an eNB may inform its neighbor(s) about which RBs will be allocated to a cell-border P2MP SL transmission in a future TTI, so that the neighboring eNB(s) avoid allocating the same resources to UL or SL transmissions. This is relatively easy to achieve, even without a central coordinator, provided that a lookahead of some TTIs is observed. With reference to Fig. 6, at $t=9$ the eNB informs its neighbor that, in a future TTI, a grant for a cell-border P2MP D2D transmission will be scheduled. The receiving eNB marks the advertised resources as unusable at the appointed TTI, and goes on with its business. The lookahead must be determined based on the delay of the X 2 connection, and can be expected to add a negligible delay to the broadcast diffusion. This mechanism is especially useful in dense networks [1], where cells are small and cell borders are traversed more frequently.

The eNBs should also select the MAC-level transmission format for P2MP transmissions. The choice of the transmission format is non-trivial, since it affects two conflicting objectives, i.e. transmission range and resource consumption. In fact, large CQI values reduce the number of RBs required for a transmission, but also decrease the transmission range on a relaying hop, thus requiring more hops to cover all the broadcasting area. On the other hand, smaller CQIs allow a single transmission to get to further UEs, hence warrant fewer hops and smaller latency. However, a transmission occupies more RBs, reducing the system capacity. Moreover, due to the frequency selectivity of the channel, using more RBs increases the probability to incur in RBs with poor conditions that may prevent a transmission to be successfully decoded. The choice of a transmission format at the $e N B$ is mandatory with SRA (since eNBs have to issue SL grants in any case, and these carry indication of the transmission format), but not so with ARS. In the latter, in fact, UEs may decide autonomously which format to use. However, we argue that the eNB is much more likely to possess a cell-wide knowledge of the UE density - which can be expected to vary much slower than the time it takes to broadcast a message - or even to know the actual UE position, to some extent, hence it should be better able to select the most suitable cell-wide transmission format. In this case, the eNB should advertise the latter periodically, using RRC procedures.

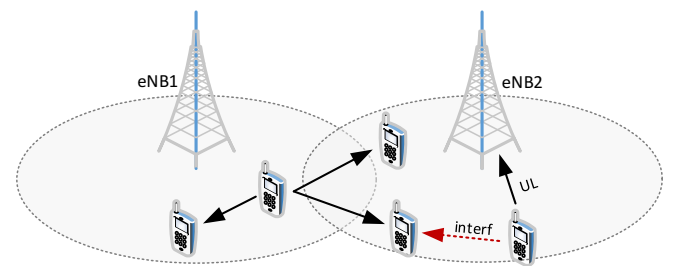

Fig. 5. Multicell scenario

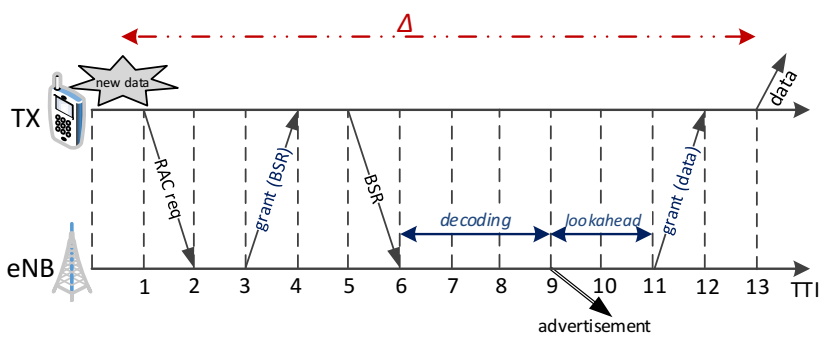

Fig. 6. SRA with coordination among eNBs

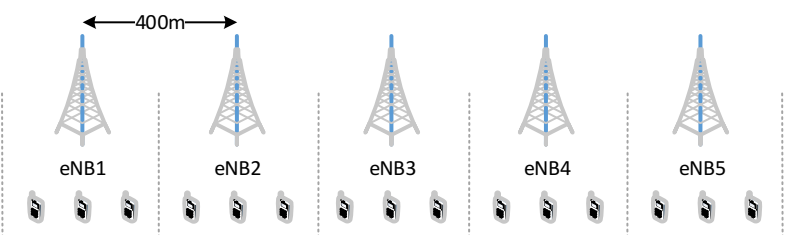

Fig. 7. Evaluation scenario

\section{Performance Evaluation}

In this section we assess the performance of multihop P2MP SL communications in terms of latency and consumed resources, comparing the different mechanisms described in Section III. Simulations are carried out using SimuLTE [9], a system-level simulator based on $\mathrm{OMNeT}++[8]$ that implements the data plane of the whole LTE-A protocol stack. We enhanced SimuLTE so as to support P2MP D2D communications. The evaluation scenario is reported in Fig. 7 and consists in five adjacent eNBs, at 400m from each other. Each eNB serves 30 UEs with P2MP SL capabilities. The latter are randomly deployed along a straight line. We assume that UEs are static and transmit at $30 \mathrm{~dB}$ in the UL and $15 \mathrm{~dB}$ in the SL. The channel is affected by Jakes fading and log-normal shadowing. Table I summarizes the main simulation parameters. A 10-byte message is generated by a random UE on each second, starting a new broadcast. In the following, we assume that UEs relay the same message only once and that the broadcast radius is $1000 \mathrm{~m}$, unless otherwise specified. 
TABLE I. MAIN SimUlation PARAMETERS

\begin{tabular}{|l|l|}
\hline \multicolumn{1}{|c|}{ Parameter } & \multicolumn{1}{c|}{ Value } \\
\hline Carrier frequency & $2 \mathrm{GHz}$ \\
\hline Bandwidth & $10 \mathrm{MHz}(50 \mathrm{RBs})$ \\
\hline Path loss model & ITU Urban Macro [11] \\
\hline Fading model & Jakes \\
\hline eNB Tx Power & $46 \mathrm{~dB}$ \\
\hline UE Tx Power & $30 \mathrm{~dB}(\mathrm{UL}) ; 15 \mathrm{~dB}(\mathrm{SL})$ \\
\hline Noise figure & $5 \mathrm{~dB}$ \\
\hline Cable loss & $2 \mathrm{~dB}$ \\
\hline Simulation time & $100 \mathrm{~s}$ \\
\hline Mobility model & Stationary \\
\hline
\end{tabular}

\section{A. Varying application-level settings}

In this subsection, we evaluate how different settings of the UE's application layer affect the performance of the broadcasting and compare multihop SL broadcasting with the relaying made by the eNB.

Fig. 8 shows which UEs receive one broadcasted message, when the broadcast area is defined using source UE's coordinates and radius, or the TTL. The message is originated by the UE denoted with the cross and should be delivered to UEs within the range defined by the vertical dashed line. Green circles indicate UEs that has received the message, whereas red squares represent UEs that have not. Explicitly defining the broadcast area (top left of Fig. 8) allows the message to reach at least all desired UEs, plus few UEs outside the range. The implementation with TTL obviously depends on how the TTL is set at the source. If the value is set to 5-6, some UEs at the border do not receive the message, whereas $\mathrm{TTL}=7$ achieves roughly the same result of defining the broadcast area. However, if the border is moved (either to the left or to the right), that TTL is no longer valid and should be modified, unlike when the broadcast area is embedded in the message.

We now evaluate the performance of multihop D2D broadcasting with and without Trickle suppression. We assume that Trickle is set such that a message is relayed if less than $K=3$ duplicates are received within a time randomly selected in the range $[5 m s, 10 m s)$. The left part of Fig. 9 shows that using Trickle slightly increases the average delay, since some UEs abstain from relaying the message and other UEs need to wait more to receive the message from a different path. On the other hand, about half the RBs are saved when Trickle is used.

We then compare our multihop solution with the one using unicast eNB relaying. In this, we assume that the source UE sends the message to the eNB only. The eNB, in turn, relays the message to the UEs under its coverage using unicast DL transmissions and to neighboring eNBs through the X2 interface. In order to define the geographical reach, we envisage two options with different levels of complexity. The simplest one is making the eNBs relay the message to all their served UEs, hence the broadcast area is a number of cells. The other solution is to allow the eNBs to inspect the application message and learn the broadcast area from it. This information can be used by the eNBs to select the subset of destination UEs, thus reducing the number of transmissions. However, this solution

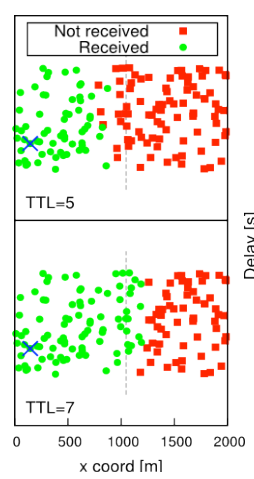

TTL

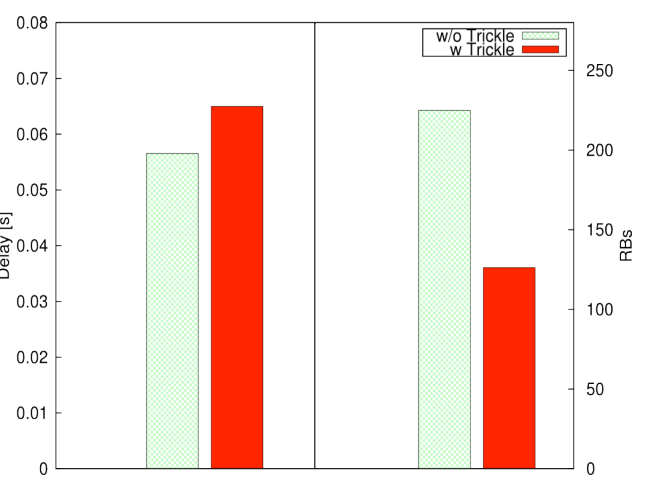

Fig. 9. Average delay (left) and average allocated RBs (right), w and w/o Trickle requires that the eNBs are enhanced with an application layer, and that they know the UE position. Fig. 10 reports the $95^{\text {th }}$ percentile of the delay and the allocated RBs in the DL subframe. As expected, delivery is faster using eNB relaying, since every UE can be reached in two hops. However, it requires around $230 \mathrm{RBs}$ to complete one broadcasting at best, whereas multihop SL does not allocate RBs in the DL.

\section{B. Varying MAC-level settings at the eNB}

Hereafter, we assume that UEs apply Trickle suppression and the broadcasting area is embedded in the message.

Fig. 11 shows how different CQI values affect the performance of a broadcast. Resources are allocated via SRA. The $\mathrm{x}$ axis reports the mean reception delay of UEs within the broadcast radius, whereas the y axis shows the mean of the total number of RBs per broadcast. Points represent the CQI. As expected, higher CQIs allow the eNBs to allocate fewer RBs. In fact, a single transmission requires eleven RBs with CQI 3 and only one RB with CQI 15. On the other hand, larger CQIs reduce the reception range and increase the number of required hops, hence increase the latency. However, we note that latency also increases with too small CQIs. As mentioned in Section III, when many RBs are used, the probability of correct reception is smaller due to fading. Thus, we can observe that CQI 7 results in the best tradeoff between latency and consumed resources. Similar results (although on different scales on both axes) are obtained for different broadcast radiuses. From now on, CQI 7 is used in the simulations.

Fig. 12 and 13 compare, respectively, the average and the $95^{\text {th }}$ percentile of the delay of SRA and ARS for different values of the broadcast radius. For ARS, we consider four different patterns, where $20 \mathrm{RBs}$ are allocated with periods 5, 10, 20 and $50 \mathrm{~ms}$ respectively. Unless large periods are used, e.g. 20-50 ms, ARS outperforms SRA, since the time spent waiting for the next transmission opportunity is smaller than the duration of a RAC handshake. However, ARS consumes much more resources, since it must reserve a predefined amount of RBs even when there is no traffic. For example, with a $20 \mathrm{~ms}$ period, one eNB must reserve 1000 RB per minute. On the other hand, SRA only allocates the required RBs, which are around 110 per broadcast over a $1000-\mathrm{m}$ radius, also factoring in BSR transmissions, as shown in Fig. 14. Fig. 15 reports the 


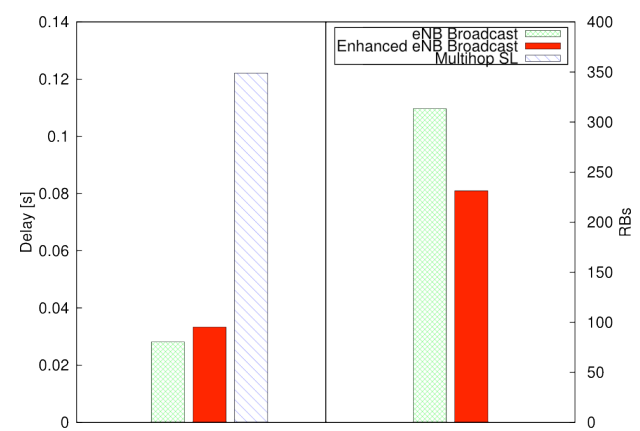

Fig. 10. $95^{\text {th }}$ percentile of delay (left) and average allocated RBs in DL (right)

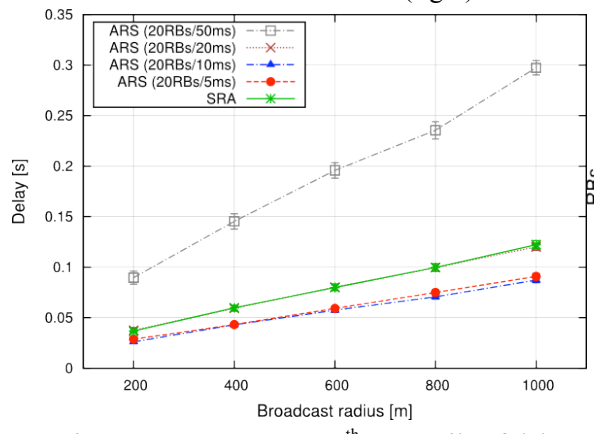

Fig. 13. SRA vs. ARS, $95^{\text {th }}$ percentile of delay

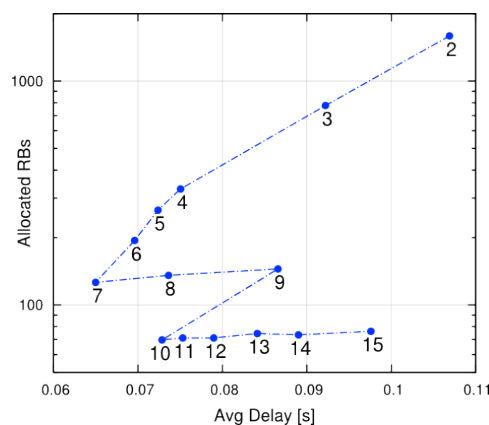

Fig. 11. Average allocated RBs and average delay

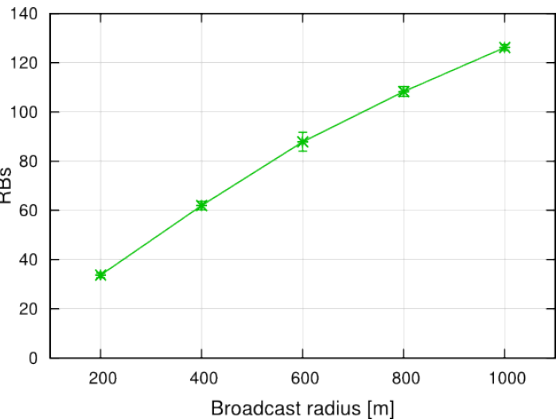

Fig. 14. SRA, avg. allocated RBs per broadcast

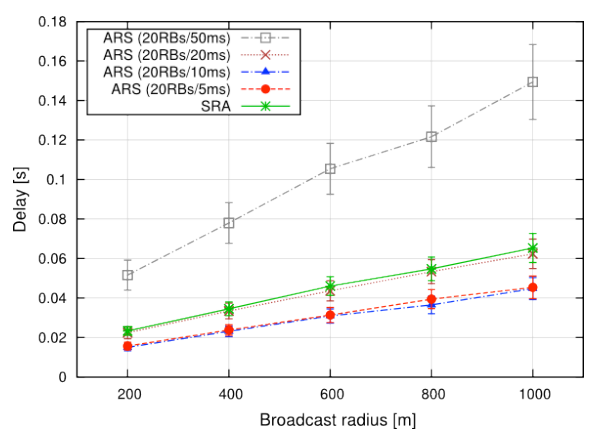

Fig. 12. SRA vs. ARS, average delay percentage of UEs that actually receive the message, which is close to $100 \%$ and fairly insensitive of the broadcast radius.

\section{CONCLUSIONS AND FUTURE WORK}

In this paper we have discussed how to broadcast messages exploiting multihop P2MP D2D transmissions. We have shown that this can be done using application-level intelligence on the UEs and standard resource allocation schemes on the eNB, and that this allows a fine-grained control over the broadcast area, which may also encompass more than a cell.

The most interesting result is that multihop D2D broadcast using scheduled resource allocation is: a) fast, i.e. $120 \mathrm{~ms}$ to cover a $1000 \mathrm{~m}$ broadcast radius at the $95^{\text {th }}$ percentile; b) reliable, and c) economical in terms of consumed resources, since around $110 \mathrm{RBs}$ per broadcast are required, i.e. less than one per reached $U E$, as well as in term of energy saving at the eNB, which stays out of data transmission. This makes such a solution viable in practice.

Future work will consider improving the broadcast by leveraging UE location information for scheduling purposes.

\section{ACKNOWLEDGMENTS}

The subject matter of this paper includes description of results of a joint research project carried out by Telecom Italia and the University of Pisa. Telecom Italia reserves all proprietary rights in any process, procedure, algorithm, article of manufacture, or other result of said project herein described.

\section{REFERENCES}

[1] L. Lei, Z. Zhong, C. Lin, X. Shen, "Operator controlled device-to-device communications in LTE-advanced networks," IEEE Wireless Comm., no. 3, pp. 96-104, June 2012
[2] C. Vallati, A. Virdis, E. Mingozzi, G. Stea, "Exploiting LTE D2D Communications in M2M Fog Platforms: Deployment and Practical Issues", Proc. of IEEE WF-IoT 2015, Milan, Italy, pp. 585-590

[3] 3GPP - TS 36.843 v12.0.1, "Study on LTE Device-to-device Proximity Services: Radio aspects (Release 12)”, March 2014

[4] X. Lin, J. Andrews, A. Ghosh, R. Ratasuk, "An overview of 3GPP deviceto-device proximity services,” IEEE Comm. Mag., 52:(4), pp.40-48, 2014

[5] P. Cappanera, L. Lenzini, A. Lori, G. Stea, G. Vaglini, "Optimal joint routing and link scheduling for real-time traffic in TDMA Wireless Mesh Networks",Computer Networks, 57(11), 2013, pp. 2301-2312

[6] R. Draves, J. Padhye, B. Zill "Routing in multi-radio, multihop wireless mesh networks", Proc. ACM Mobicom'04, Philadelphia, US pp. 114-128

[7] P. Levis, N. Patel, D. Culler and S. Shenker, "Trickle: A self-regulating algorithm for code propagation and maintenance in wireless sensor networks", Proc. 1st USENIX/ACM Symp. NSDI, pp. 15-28, 2004

[8] A. Varga, R. Hornig, "An overview of the OMNeT++ simulation environment", in Proc. SIMUTools '08, Marseille, FR, March 2008.

[9] A. Virdis, G. Stea, G. Nardini, "Simulating LTE/LTE-Advanced Networks with SimuLTE", DOI 10.1007/978-3-319-26470-7_5, in: Advances in Intelligent Systems and Computing, Vol. 402, pp. 83-105, Springer

[10] G. Nardini, G. Stea, A. Virdis, D. Sabella, M. Caretti, "Resource allocation for network-controlled device-to-device communications in LTEAdvanced", Wireless Networks, 2017, DOI: 10.1007/s11276-016-1193-3

[11] 3GPP TR 36.814 v9.0.0, "Further advancements for E-UTRA physical layer aspects (Release 9)," March 2010

[12] B. Williams, T. Camp, "Comparison of broadcasting techniques for mobile ad hoc networks," Proc. of MOBIHOC '02, Lausanne, CH, 2002

[13] P. Kyasanur, R. R. Choudhury, I. Gupta, "Smart Gossip: An Adaptive Gossip-based Broadcasting Service for Sensor Networks," Proc. IEEE MASS 2006, Vancouver, BC, pp. 91-100.

[14] A. Cimmino et al. "The Role of Small Cell Technology in Future Smart City Applications", Trans. on Emerging Telecom. Tech. 25 (1), 11-20

[15] J. M. B. da Silva Jr, G. Fodor, T.F. Maciel, "Performance Analysis of Network-Assisted Two-Hop D2D Communications", Proc. Globecom 2014, Austin (TX), 8-12 Dec. 2014

[16] Siyi Wang, et al., "Outage Probability for Multi-Hop D2D Communications With Shortest Path Routing”, IEEE Comm. Letters, 19(11), Nov. 2015

[17] G Rigazzi, et al., "Multi-hop D2D networking and resource management scheme for M2M communications over LTE-A systems", Proc. IEEE IWCMC '14, Nicosia (CY), 4-8 Aug. 2014 